\title{
Deep Learning Based Smart Survilance Robot
}

\author{
Dr.Vithya Ganesan ${ }^{1}$, Smritilekha Das ${ }^{2}$,Tamal Kumar Kundu ${ }^{3}$,Naren.J ${ }^{4}$ Dr.S.Nikkath Bushra ${ }^{5}$ \\ vithyamtech@gmail.com¹,dassmritilekha007@gmail.com²,kundutamal007@gmail.com³ \\ Professor $^{1}$,Asst Professor ${ }^{2}$, Research Scholar ${ }^{3}$,CSE Dept,Koneru Lakshmaiah Education \\ Foundation, Green Fields, Vaddeswaram, Guntur District-522502, Andhra Pradesh, Research \\ Scholar ${ }^{4}$,India,Asso Professor ${ }^{5}$,IT Dept, St.Joseph's Institute of Technology,Chennai, 119
}

\begin{abstract}
Surveillance Robot aims to blend IoT capabilities with the support of cloud and machine learning is an advancement to deliver a sophisticated solution for real time security. Industrial and commercial surveillance data security is required for small camera as well as large scale deployment with a drones or robot cars. This paper deals with face recognition using AWS Rekognition and video streaming using AWS kinesis and AWS SNS(Simple notification Service). AWS Rekognition uses deep learning algorithms to introspect the video stream and find objects / faces on them and compare it with the collection of information that it has trained previously. It detects face with video feed and scans the database to identify the person with AWS Rekognition, there is also an option of adding new faces by uploading photo of the person to an S3 bucket and face can be indexed .
\end{abstract}

Keywords:Surveillance Robot, AWS Rekognition, AWS kinesis

\section{Introduction}

Internet of Things (IoT) fasten with Robotics in real time fields such as Industry surveillance, superintendence of skilling worker in connection with video, image data is a challanged work for smart attendance management with face detection, sentiment analysis ,Face based user verifications .With the suppor of Amazon Rekognition,Facial analysis and search is used to identify andexamine user verification,counting faces, and also for public safety. As well as it support tagging and indexing of photos as a service with scaling infrastructure.Visual search, tag-based browsing and discovery models are reinforced by Amazon Rekognition. AWS Rekognition contributes in validatting authentication and security. For example, in artisan attendance bio metric system, authentication has been done for entering into secure place by comparing the stored employee images with real time camera images and giving a sign before allowing an employee to enter in to secured zone. 
AWS SNS with details of known and unknown person in the video acts as a message broker and forwards the notification to Google cloud messaging subscriber ,an android application running on a phone. A notification will be popped up on the android mobile phone running the application with a picture of the person detected in the video feed.

Real time process,analyze and streaming data in short time processing is by Amazon Kinesis with cost-effectively process .Video, audiologs, URL click streams, and IoT telemetry data uses kinesis by machine learning algorithm . Amazon Kinesis process and analyze data quickly so, waiting time will be less .

Amazon Rekognition, identify the images and videos and get facial attributes such as skin tone, texure, eye color, age and facial hair which are used to measure changes based on constructing a timeline of the emotions expressed by an action

The paper is divided in 4 parts. First, using Deep Learning based Smart surveillance. Second, working methodology and a platform for reads and transmits the data to android based application. Third, creation of AWS Rekognition for data collection and storing . Fourth ,AWS Kinesis analyzing video to get contextual data. Working methodolgy results, and future enhancements are discussed at the end of the sections.

\section{2 . Literature Review}

Quality of Experince(QoE) for Video gushing in a distributed environment is encoded by an erasure code.[1],[2]. A customization or contextual system is required to dectect event based or semantic based video anaysis with less computational power and hardware. In cloud, Customized or contextual video surveillances analysed by cloud based object detection.It is by You Only Look Once (YOLO) object detection model, which identifies the objects within the frame[3],[4].

Real-time Video survillience is more rational to increase communal safety, e-commerce and health technology support commutations . In the existing technologies of cloud the video analytics has been done by preloaded video data and which is to from centralized cluster or the cloud. Most of the time is wasting in transmission and latency, which leads to high cost of video data transmissions between IoE devices and cloud [5]. Computer vision is in need to pecularities in video processing of public safety. High detection and accuracy of video by 
Deep Neural Networks (i.e., DNNs) can be used for skinning layered video with less amount of computing power and storage space. Thus, DNNs-based video analytics service is required in the cloud to perusal the stationary cameras [6],[7][8].

The main motivation of this work is to identify the pitfalls in cloud video synthesis to process large-volume of data to avoid latency in critical applications[11][12]. Cloud computing aims enormous resources that can be flexibly used to fit dynamic demands which leads to avoid substantial latencies when using services placed at distant locations [9],[10]

\title{
3.WORKING PRINCIPLE
}

There are Three Different Sections to be discussed

\author{
1. AWS Rekognition \\ 2,AWS kinesis \\ 3. AWS SNS
}

Figure 1 explain the pin configuration of camera robot 


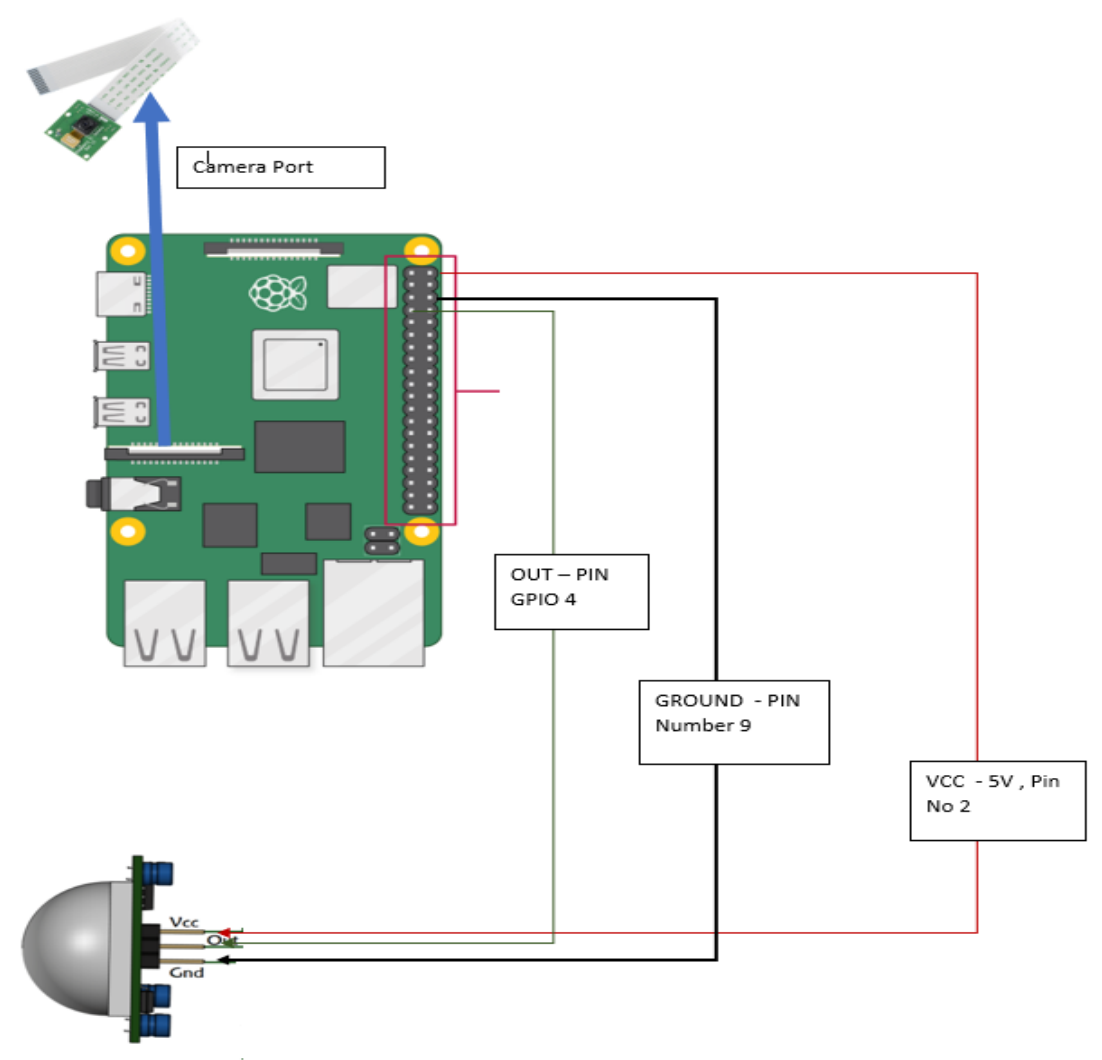

Fig. 1. Pin configuration of Camera Robot

\subsection{AWS Rekognition}

To recognize faces in the video streams using Amazon Rekognition Video, or process it for live streaming. It detects face with video feed and scans the database to identify the person with AWS. AWS Rekognition uses deep learning algorithms to interrospect the video stream and find objects / faces on them and compare it with the collection of information that it has already learned previously. 
Creating a collection and make the engine learn an image:

- aws rekognition create-collection

--collection-id

surveilance_bot_collection --region us-west-2

- aws rekognition index-faces --image

'\{"S3Object": \{"Bucket":"shefeekjbits","Name":"shefeek.jpg"\}\}' -collection-id "surveilance_bot_collection" --detection-attributes "ALL" --external-image-id "Shefeek" --region us-west-2

To start with AWS Rekognition, a cloud formation stack need to be created with all the required components.AWS provide pre provisioned stack that can be used directly and explained in Figure 2.

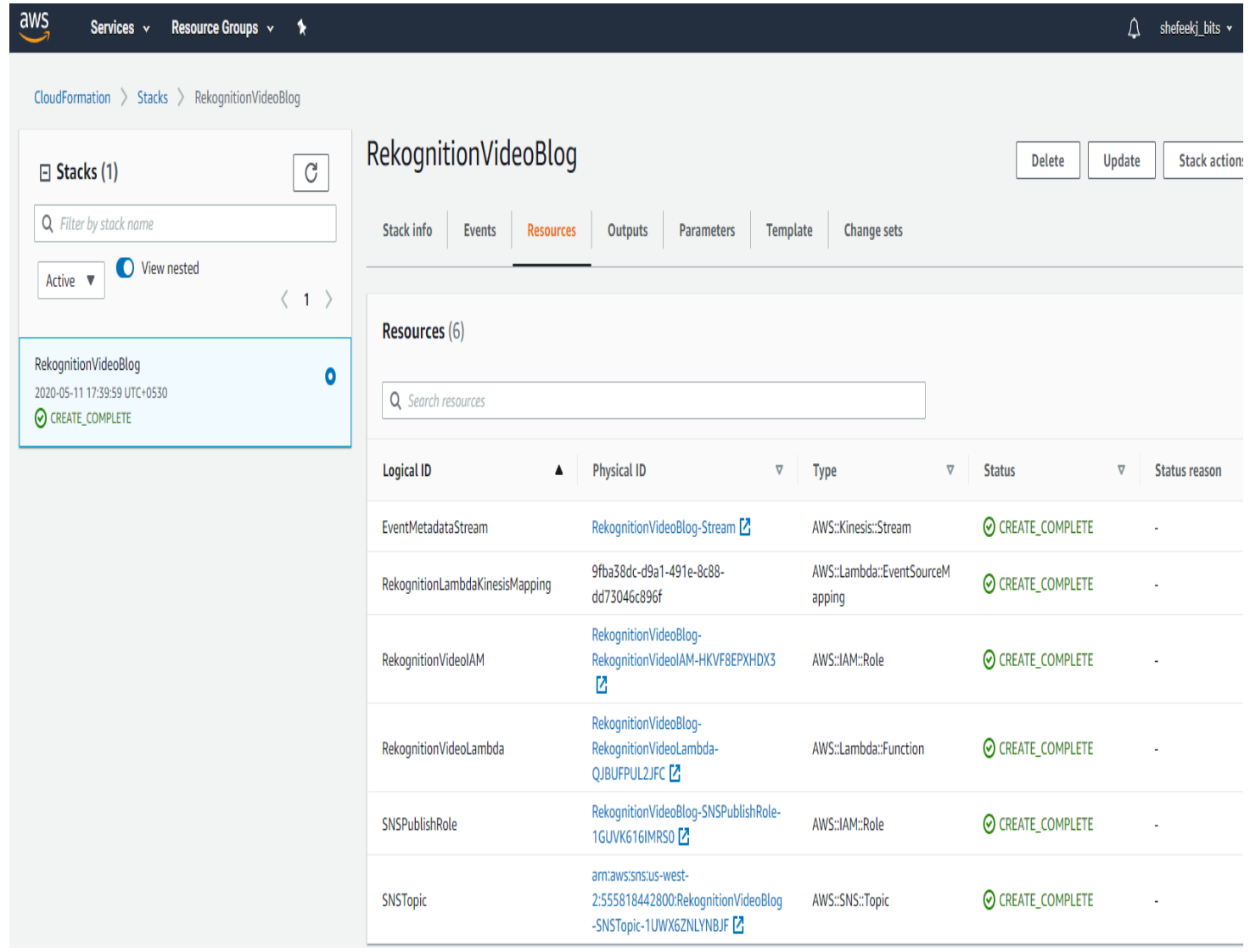

Fig: 2. Creation of AWS Rekognition 


\subsection{AWS KINESIS}

Kinesis video stream is created on AWS by Kinesis Video Producer libraries need to be downloaded (CPP) and compiled on the target machine operating system ( Raspbian ). This library enables to stream videos from the device to a kinesis video stream.

Stream video from a Raspberry Pi (with camera module) to AWS using Kinesis Video Streams for securly stream video. In AWS Rekognition there is also an option of adding new faces by uploading photo of the person to an S3 bucket and face can be indexed and shown in Figure 3.

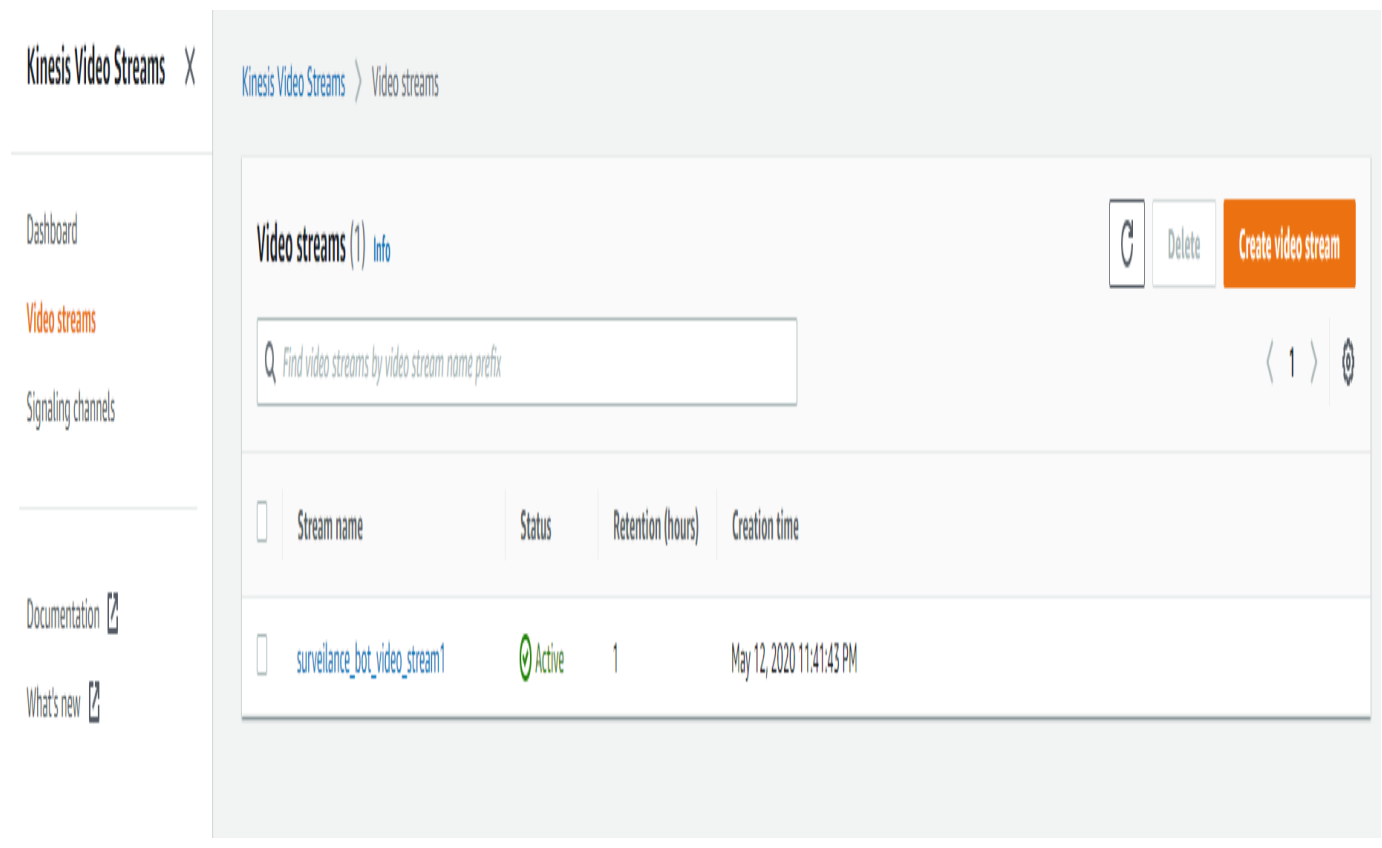

Fig 3. Kinesis Video Stream

AWS SNS with details of known and unknown person in the video.AWS SNS acts as a message broker and forwards the notification to Google cloud messaging subscriber ,an android application running on a phone. A notification will be popped up on the android mobile phone running the application with a picture of the person detected in the video feed shoes in Figure 4. 


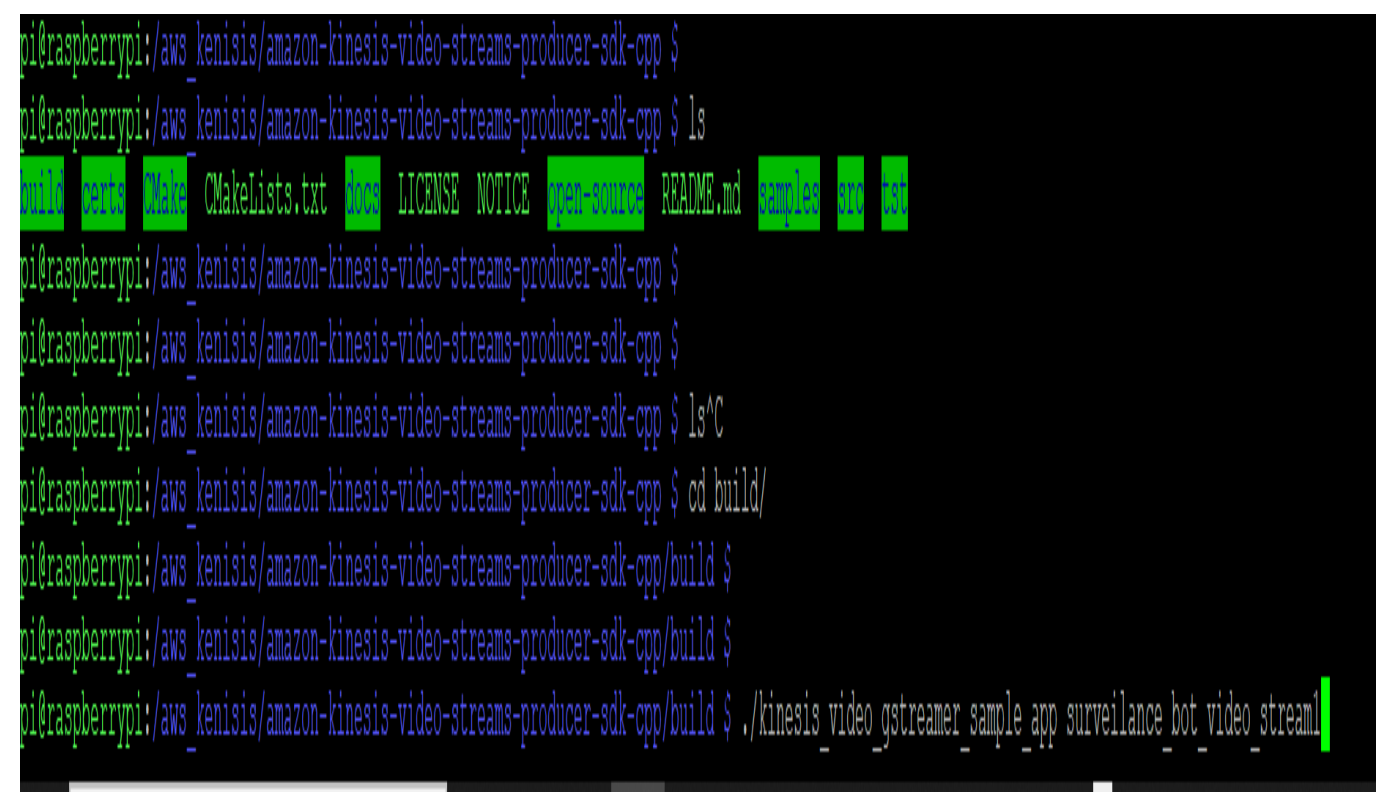

Fig 4. Video streaming from Rasberrypi

An AWS Rekognition video stream processor need to be created and integrated with kinesis video stream with the following command

aws rekognition create-stream-processor --region us-east-2 --cli-input-json file:///home/pi/aws_rekognition/cli-input.json

Cli-input - https://shefeekjbitss3.s3-us-west-amazonaws.com/cli-input.json

Once created, it can be started by

aws rekognition start-stream-processor --name <stream_processor_name $>$

\section{3AWS SNS(Amazon simple notification Service)}

Notification Push is delivered to a platform application (Android application ). An android project is created in Google FireBase, an application development platform and the registration token is used to develop a simple android application that has two features

- Show the token of the application installation instance .Each device which installs the application will be assigned a unique token by Google FireBase. 
- Show notification on to the phone as and when it receives one from google firebase cloud messaging service.

AWS Simple Notification Service( SNS ) is created and is integrated with AWS Rekognition to ensure that a notification message is received by sns whenever a known or unknown face is detected by aws rekognition.SNS acts as a message broker and aws rekognition acts as a message publisher. Android devices will be added as subscribers on to SNS with the token generated on the android device and it is shown in Figure 5.

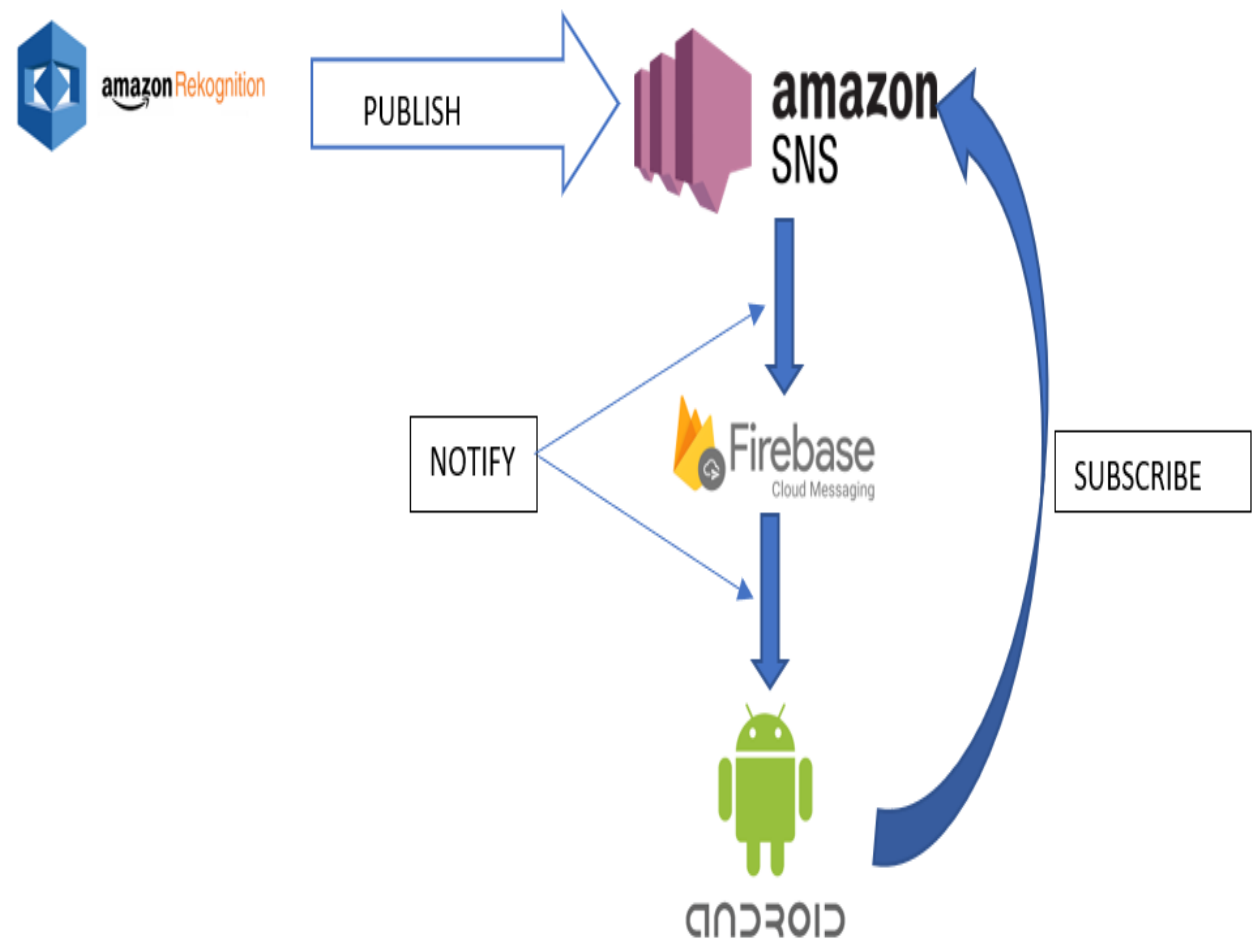

Fig 5. Android project in Google FireBase 


\section{Conclusion and future Enhancement}

AWS Rekognition and Amazon Kinesis relate accurate facial analysis by detecting facial identification marks. It is useful for comparing faces, wide variety of user verification, people counting, and widespread safety by using AWS. Rekognition ,Amazon Kinesis suits well for process and analyze real-time, streaming data within shorter duration of time .It can get timely insights and react quickly to accord new information. video streaming analysis identifies the person in the video by comparing the database which contains already trained data, it also identifies the new persons which is not trained using the concept of deep learning.

As a future enhancement convolutional neural network $(\mathrm{CNN})$ can be used for facial recognition in live video streaming without training the network. it can learn by itself when a new person arrives within a fraction of second. The computational time can be reduced to a grater extent.

\section{REFERENCES}

[1] Abubakr O. Al-Abbasi, Vaneet Aggarwal, "Video Streaming in Distributed Erasure-Coded StorageSystems: Stall Duration Analysis", Networking IEEE/ACM Transactions on, vol. 26, no. 4, pp. 1921-1932, 2018

[2] S. Choi, E. Salter, X. Zhang and B. C. Wünsche, "Bird Eyes: A Cloud-Based Object Detection System for Customisable Surveillance," 2018 International Conference on Image and Vision Computing New Zealand (IVCNZ), Auckland, New Zealand, 2018, pp. 1-6, doi: 10.1109/IVCNZ.2018.8634751.

[3] Q. Zhang, Q. Zhang, W. Shi and H. Zhong, "Firework: Data Processing and Sharing for Hybrid Cloud-Edge Analytics," in IEEE Transactions on Parallel and Distributed Systems, vol. 29, no. 9, pp. 2004-2017, Sept. 2018, doi: 10.1109/TPDS.2018.2812177.

[4] Si Young Jang, Yoonhyung Lee, Byoungheon Shin, Dongman Lee, "Application-Aware IoT Camera Virtualization for Video Analytics Edge Computing", Edge Computing (SEC) 2018 IEEE/ACM Symposium on, pp. 132-144, 2018.

[5] Qingyang Zhang, Hui Sun, Xiaopei Wu, Hong Zhong, "Edge Video Analytics for Public Safety: A Review", Proceedings of the IEEE, vol. 107, no. 8, pp. 1675-1696, 2019.

[6] An Wang, Aziz Mohaisen, Songqing Chen, "XLF: A Cross-layer Framework to Secure the Internet of Things (IoT)", Distributed Computing Systems (ICDCS) 2019 IEEE 39th International Conference on, pp. 1830-1839, 2019.

[7] Hui Sun, Ying Yu, Kewei Sha, Bendong Lou, "mVideo: Edge Computing Based Mobile Video Processing Systems", Access IEEE, vol. 8, pp. 11615-11623, 2020.

[8] Q. Zhang, Q. Zhang, W. Shi, and H. Zhong, "Firework: Data processing and sharing for hybrid cloud-edge analytics," IEEE Trans. Parallel Distrib. Syst., vol. 29, no. 9, pp. 2004 2017, Sep. 2018.

[9] Amazon .AWS DeepLens, https://aws.amazon.com/deeplens/ (2018, accessed 19 January 2019). 
[10] J. Gedeon, F. Brandherm, R. Egert, T. Grube and M. Mühlhäuser, "What the Fog? Edge Computing Revisited: Promises, Applications and Future Challenges," in IEEE Access, vol. 7, pp. 152847-152878, 2019, doi: 10.1109/ACCESS.2019.2948399.

[11] M. Ananthi, K. Vijayakumar , "An intelligent approach for dynamic network traffic restriction using MAC address verification”, Computer Communications,Elesvier,5 February 2020.

[12] K. Pradeep Mohan Kumar, M. Saravanan, M. Thenmozhi ,K. Vijayakumar, “ Intrusion detection system based on GA-fuzzy classifier for detecting malicious attacks", wiley, Feb 2019. 